\title{
One-pot Multi-step Bohlmann-Rahtz Heteroannulation Reactions: Synthesis of Dimethyl Sulfomycinamate
}

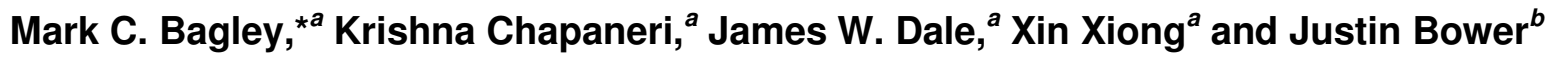 \\ ${ }^{a}$ Cardiff School of Chemistry, Cardiff University, PO Box 912, Cardiff, CF10 3TB, UK. \\ ${ }^{b}$ Vernalis, Granta Park, Abington, Cambridge, CB1 6GB, UK. \\ Email AddressBagleymc@cf.ac.uk
}

\section{CONTENTS.}

S1 General Procedures

S2 Characterisation Data for Pyridines $\mathbf{1 5}$

S5 ${ }^{1} \mathrm{H}$ NMR Spectra of Intermediates in the Synthesis of Dimethyl Sulfomycinamate

S11 ${ }^{1}$ H NMR Spectrum of Dimethyl Sulfomycinamate (5)

S12 ${ }^{13}$ C NMR Spectrum of Dimethyl Sulfomycinamate (5)

General Procedures. Commercially available reagents were used without further purification; solvents were dried by standard procedures. Light petroleum refers to the fraction with bp $40-60{ }^{\circ} \mathrm{C}$ and ether refers to diethyl ether. Flash chromatography was carried out using Merck Kieselgel $60 \mathrm{H}$ silica or Matrex silica 60. Analytical thin layer chromatography was carried out using aluminium-backed plates coated with Merck Kieselgel $60 \mathrm{GF}_{254}$ that were visualised under UV light (at 254 and/or 360 $\mathrm{nm})$. Microwave irradiation experiments were performed using a self-tunable CEM Discover ${ }^{\mathrm{TM}}$ focused monomodal microwave synthesiser at the given temperature by varying the irradiation power (initial power given in parentheses). Infra-red (IR) spectra were recorded in the range 4000-600 $\mathrm{cm}^{-1} \mathrm{using}$ $\mathrm{KBr}$ disks for solid samples and thin films between $\mathrm{NaCl}$ plates for liquid samples or as a nujol mull and are reported in $\mathrm{cm}^{-1}$. Nuclear magnetic resonance (NMR) spectra were recorded in $\mathrm{CDCl}_{3}$ at $25{ }^{\circ} \mathrm{C}$ unless stated otherwise and were reported in ppm; $J$ values were recorded in $\mathrm{Hz}$ and multiplicities were expressed by the usual conventions. Low-resolution mass spectra (MS) were determined using atmospheric pressure chemical ionization (APcI) unless otherwise stated. ES refers to electrospray ionization, CI refers to chemical ionization (ammonia) and EI refers to electron ionization. Highresolution mass spectra were obtained courtesy of the EPSRC Mass Spectrometry Service at University College of Wales, Swansea, UK using the ionization methods specified. In vacuo refers to evaporation at reduced pressure using a rotary evaporator and diaphragm pump, followed by the removal of trace volatiles using a vacuum (oil) pump. 
Ethyl 2,6-Dimethylpyridine-3-carboxylate (15aa). The title compound ${ }^{1,2}$ was obtained as a pale yellow oil (Found: $\mathrm{MH}^{+}, 180.1024 . \mathrm{C}_{10} \mathrm{H}_{13} \mathrm{NO}_{2}$ requires $\mathrm{MH}^{+}, 180.1021$ ); IR (film) $v_{\max } 2983,1724$, 1593, 1446, 772; ${ }^{1} \mathrm{H}$ NMR (400 MHz; $\left.\mathrm{CDCl}_{3}\right) \delta 8.03(1 \mathrm{H}, \mathrm{d}, J$ 8.0), 6.99 (1H, d, J 8.0), 4.29 (2H, q, J 7.1), $2.74(3 \mathrm{H}, \mathrm{s}), 2.50(3 \mathrm{H}, \mathrm{s}), 1.32(3 \mathrm{H}, \mathrm{t}, J 7.1) ;{ }^{13} \mathrm{C} \mathrm{NMR}\left(100 \mathrm{MHz} ; \mathrm{CDCl}_{3}\right) \delta 167.1(\mathrm{C}), 161.6(\mathrm{C})$, $159.8(\mathrm{C}), 139.2(\mathrm{CH}), 123.1(\mathrm{C}), 120.8(\mathrm{CH}), 61.4\left(\mathrm{CH}_{2}\right), 25.2(\mathrm{Me}), 25.1(\mathrm{Me}), 14.7(\mathrm{Me})$; MS (APcI) $m / z 180\left(\mathrm{MH}^{+}, 100 \%\right)$.

Ethyl 2,6-Dimethyl-4-ethylpyridine-3-carboxylate (15ab). The title compound ${ }^{2}$ was obtained as a pale yellow oil (Found: $\mathrm{MH}^{+}$, 208.1337. $\mathrm{C}_{12} \mathrm{H}_{17} \mathrm{NO}_{2}$ requires $M H^{+}$, 208.1335); IR (film) $v_{\max } 2975$, 2937, 1726, 1595, 1561, 1190, 1089; ${ }^{1} \mathrm{H}$ NMR (400 MHz, $\left.\mathrm{CDCl}_{3}\right) \delta 6.85$ (1H, s), 4.32 (2H, q, J 7.1), $2.50\left(2 \mathrm{H}, \mathrm{q}, J\right.$ 7.6), $2.44(3 \mathrm{H}, \mathrm{s}), 2.42(3 \mathrm{H}, \mathrm{s}), 1.30(3 \mathrm{H}, \mathrm{t}, J 7.1), 1.12(3 \mathrm{H}, \mathrm{t}, J 7.6) ;{ }^{13} \mathrm{C}$ NMR (100 $\left.\mathrm{MHz}, \mathrm{CDCl}_{3}\right) \delta 169.5(\mathrm{C}), 158.9(\mathrm{C}), 154.7(\mathrm{C}), 151.1(\mathrm{C}), 126.7(\mathrm{C}), 120.7(\mathrm{CH}), 61.6\left(\mathrm{CH}_{2}\right), 26.6$ $\left(\mathrm{CH}_{2}\right), 24.7(\mathrm{Me}), 23.2(\mathrm{Me}), 15.0(\mathrm{Me}), 14.5(\mathrm{Me})$; MS (APcI) m/z (relative intensity) $208\left(\mathrm{MH}^{+}\right.$, $100 \%)$.

Ethyl 2,6-Dimethyl-4-phenylpyridine-3-carboxylate (15ac). The title compound ${ }^{2}$ was obtained as a pale yellow oil (Found: $\mathrm{MH}^{+}, 256.1337 . \mathrm{C}_{16} \mathrm{H}_{17} \mathrm{NO}_{2}$ requires $M H^{+}, 256.1334$ ); IR (film) $v_{\max } 2978$, 2926, 1725, 1587, 1548, 1266, 1206, 1083, 870, 767, 701; ${ }^{1} \mathrm{H}$ NMR (400 MHz, $\left.\mathrm{CDCl}_{3}\right) \delta 7.30(5 \mathrm{H})$, $6.94(1 \mathrm{H}, \mathrm{s}), 4.01\left(2 \mathrm{H}, \mathrm{q}, J\right.$ 7.1), $2.54(3 \mathrm{H}, \mathrm{s}), 2.50(3 \mathrm{H}, \mathrm{s}), 0.90(3 \mathrm{H}, \mathrm{t}, J 7.1) ;{ }^{13} \mathrm{C} \mathrm{NMR}(100 \mathrm{MHz}$, $\left.\mathrm{CDCl}_{3}\right) \delta 169.5(\mathrm{C}), 159.1(\mathrm{C}), 155.5(\mathrm{C}), 148.9(\mathrm{C}), 139.2(\mathrm{C}), 128.9(\mathrm{CH}), 128.8(\mathrm{CH}), 128.2(\mathrm{CH})$, $126.1(\mathrm{C}), 121.6(\mathrm{CH}), 61.7\left(\mathrm{CH}_{2}\right), 24.9(\mathrm{Me}), 23.2(\mathrm{Me}), 14.0(\mathrm{Me}) ; \mathrm{MS}(\mathrm{APcI}) m / z$ (relative intensity) $256\left(\mathrm{MH}^{+}, 100 \%\right), 252(2)$.

Ethyl 2-Methyl-6-phenylpyridine-3-carboxylate (15ad). The title compound was obtained as a pale yellow solid, mp 44-45 ${ }^{\circ} \mathrm{C}(\mathrm{MeOH})$ (lit. ${ }^{1} \mathrm{mp} 44{ }^{\circ} \mathrm{C}$ ) (Found: $\mathrm{C}, 74.4 ; \mathrm{H}, 6.5 ; \mathrm{N}, 5.6$. Calc. for $\mathrm{C}_{15} \mathrm{H}_{15} \mathrm{NO}_{2}$ : C, C, 74.7; H, 6.3; N, 5.8\%) (Found: $\mathrm{MH}^{+}$, 242.1182. $\mathrm{C}_{15} \mathrm{H}_{15} \mathrm{NO}_{2}$ requires $M H, 242.1182$ ); IR (KBr) $v_{\max } 2980,2925,2890,1717,1581,1476,1277,1090,1022 ;{ }^{1} \mathrm{H}$ NMR (400 MHz, $\left.\mathrm{CDCl}_{3}\right) \delta$ $8.19(1 \mathrm{H}, \mathrm{d}, J 8.2), 8.00(2 \mathrm{H}, \mathrm{m}), 7.55(1 \mathrm{H}, \mathrm{d}, J 8.2), 7.41(3 \mathrm{H}), 4.33(2 \mathrm{H}, \mathrm{q}, J 7.1), 2.85(3 \mathrm{H}, \mathrm{s}), 1.35$ $\left(3 \mathrm{H}, \mathrm{t}, J\right.$ 7.1); ${ }^{13} \mathrm{C}$ NMR (100 MHz, $\left.\mathrm{CDCl}_{3}\right) \delta 165.7$ (C), 159.1 (C), $158.1(\mathrm{C}), 138.5(\mathrm{CH}), 137.6(\mathrm{C})$, $128.7(\mathrm{CH}), 128.0(\mathrm{CH}), 126.5(\mathrm{CH}), 122.8(\mathrm{C}), 116.5(\mathrm{CH}), 60.3\left(\mathrm{CH}_{2}\right), 24.6(\mathrm{Me}), 13.5(\mathrm{Me})$; MS (APcI) $m / z$ (relative intensity) 241 (M+, 91\%), 240 (69), 212 (32), 196 (100), 195 (98), 168 (43), 167 (40).

\footnotetext{
${ }^{1}$ Bohlmann, F.; Rahtz, D. Chem. Ber. 1957, 90, 2265.

${ }^{2}$ Bagley, M. C.; Brace, C.; Dale, J. W.; Ohnesorge, M.; Phillips, N. G.; Xiong, X.; Bower, J. J. Chem. Soc., Perkin Trans. 1 2002, 1663.
} 
Ethyl 2-Methyl-6-(4-chlorophenyl)pyridine-3-carboxylate (15ae). The title compound was obtained as a pale yellow solid, mp $47-48{ }^{\circ} \mathrm{C}$ (aqueous EtOH) (lit. ${ }^{3} \mathrm{mp} 47-48{ }^{\circ} \mathrm{C}$ ) (Found: $\mathrm{M}^{+}$, 275.0714. $\mathrm{C}_{15} \mathrm{H}_{14}{ }^{35} \mathrm{ClNO}_{2}$ requires $\left.M^{+}, 275.0713\right)$; IR (KBr) $v_{\max } 2982,1719,1585,1456,1270,1093$, 1012, 834, 784; ${ }^{1} \mathrm{H}$ NMR (400 MHz, $\left.\mathrm{CDCl}_{3}\right) \delta 8.19(1 \mathrm{H}, \mathrm{d}, J 8.2), 7.94(2 \mathrm{H}, \mathrm{d}, J 8.6), 7.52(1 \mathrm{H}, \mathrm{d}, J$ 8.2), $7.37\left(2 \mathrm{H}, \mathrm{d}, J\right.$ 8.6), $4.32(2 \mathrm{H}, \mathrm{q}, J 7.1), 2.83(3 \mathrm{H}, \mathrm{s}), 1.34(3 \mathrm{H}, \mathrm{t}, J 7.1) ;{ }^{13} \mathrm{C} \mathrm{NMR}(100 \mathrm{MHz}$, $\left.\mathrm{CDCl}_{3}\right) \delta 166.9(\mathrm{C}), 160.4(\mathrm{C}), 158.1(\mathrm{C}), 139.8(\mathrm{CH}), 137.2(\mathrm{C}), 136.3(\mathrm{C}), 129.4(\mathrm{CH}), 129.0(\mathrm{CH})$, $124.3(\mathrm{C}), 117.5(\mathrm{CH}), 61.6\left(\mathrm{CH}_{2}\right), 25.7(\mathrm{Me}), 14.7(\mathrm{Me})$; MS (APcI) $m / z$ (relative intensity) $278\left(\mathrm{MH}^{+}\right.$, $25 \%), 276\left(\mathrm{MH}^{+}, 100 \%\right), 150(10)$.

Ethyl 2-methyl-6-(4-methoxyphenyl)pyridine-3-carboxylate (15af). The title compound was obtained as a pale yellow solid, mp 68-69 ${ }^{\circ} \mathrm{C}(\mathrm{MeOH})$ (lit. ${ }^{4} \mathrm{mp} 68-69{ }^{\circ} \mathrm{C}$ ) (Found: $\mathrm{M}^{+}, 271.1206$. $\mathrm{C}_{16} \mathrm{H}_{17} \mathrm{NO}_{3}$ requires $\left.M^{+}, 271.1208\right)$; IR (KBr) $v_{\max } 2979,2933,2835,1717,1584,1514,1460,1269$, 1187, 1094, 1036, 828, 786; ${ }^{1} \mathrm{H}$ NMR (400 MHz, $\left.\mathrm{CDCl}_{3}\right) \delta 8.15$ (1H, d, J 8.2), $7.96(2 \mathrm{H}, \mathrm{d}, J$ 8.8), 7.48 $\left(1 \mathrm{H}, \mathrm{d}, J\right.$ 8.2), $6.92\left(2 \mathrm{H}, \mathrm{d}, J\right.$ 8.8), $4.31(2 \mathrm{H}, \mathrm{q}, J 7.1), 3.79(3 \mathrm{H}, \mathrm{s}), 2.82(3 \mathrm{H}, \mathrm{s}), 1.34(3 \mathrm{H}, \mathrm{t}, J 7.1) ;{ }^{13} \mathrm{C}$ NMR (100 MHz, $\left.\mathrm{CDCl}_{3}\right) \delta 167.2$ (C), 161.4 (C), 160.3 (C), 159.1 (C), 139.7 (CH), 131.5 (C), 129.1 $(\mathrm{CH}), 123.2(\mathrm{C}), 116.9(\mathrm{CH}), 114.6(\mathrm{CH}), 61.5\left(\mathrm{CH}_{2}\right), 55.8(\mathrm{Me}), 25.8(\mathrm{Me}), 14.7(\mathrm{Me})$; MS (APcI) $m / z$ (relative intensity) $272\left(\mathrm{MH}^{+}, 100 \%\right), 150(10)$.

Ethyl 2,4-Diphenyl-6-methylpyridine-3-carboxylate (15bc). The title compound ${ }^{2}$ was obtained as a colorless solid, mp 99-100 ${ }^{\circ} \mathrm{C}$ (light petroleum) (Found: $\mathrm{C}, 79.5 ; \mathrm{H}, 6.0 ; \mathrm{N}, 4.4 . \mathrm{C}_{21} \mathrm{H}_{19} \mathrm{NO}_{2}$ requires C, 79.9; H, 6.3; N, 4.4\%) (Found: $\mathrm{MH}^{+}$, 318.1494. $\mathrm{C}_{21} \mathrm{H}_{19} \mathrm{NO}_{2}$ requires $M H, 318.1496$ ); IR (nujol) $v_{\max }$ 2928, 1722, 1543, 1269, 1205, 1110, 1053, 770, 746, 701; ${ }^{1} \mathrm{H}$ NMR (400 MHz, $\left.\mathrm{CDCl}_{3}\right) \delta 7.53(2 \mathrm{H}, \mathrm{m})$, $7.30(8 \mathrm{H}), 7.07(1 \mathrm{H}, \mathrm{s}), 3.83(2 \mathrm{H}, \mathrm{q}, J 7.2), 2.57(3 \mathrm{H}, \mathrm{s}), 0.75(3 \mathrm{H}, \mathrm{t}, J 7.2) ;{ }^{13} \mathrm{C} \mathrm{NMR}(100 \mathrm{MHz}$, $\left.\mathrm{CDCl}_{3}\right) \delta 169.7(\mathrm{C}), 157.9(\mathrm{C}), 155.5(\mathrm{C}), 147.8(\mathrm{C}), 138.9(\mathrm{C}), 137.3(\mathrm{C}), 127.5(\mathrm{CH}), 127.4(\mathrm{CH})$, $127.4(\mathrm{CH}), 127.3(\mathrm{CH}), 127.0(\mathrm{CH}), 124.9(\mathrm{C}), 121.2(\mathrm{CH}), 60.2\left(\mathrm{CH}_{2}\right), 23.6(\mathrm{Me}), 12.4(\mathrm{Me}) ; \mathrm{MS}$ (APcI) $m / z$ (relative intensity) $318\left(\mathrm{MH}^{+}, 100 \%\right)$.

tert-Butyl 2,6-Dimethylpyridine-3-carboxylate (15ca). The title compound ${ }^{2}$ was obtained as a pale yellow oil (Found: $\mathrm{MH}^{+}, 208.1337 . \mathrm{C}_{12} \mathrm{H}_{17} \mathrm{NO}_{2}$ requires $M H, 208.1337$ ); IR (film) $v_{\max } 2977,2930$, 2856, 1720, 1592, 1568, 1127, 1083. ${ }^{1} \mathrm{H} \mathrm{NMR}\left(400 \mathrm{MHz}, \mathrm{CDCl}_{3}\right) \delta 7.95$ (1H, d, J 8.0), $6.97(1 \mathrm{H}, \mathrm{d}, J$ 8.0), $2.71(3 \mathrm{H}, \mathrm{s}), 2.51(3 \mathrm{H}, \mathrm{s}), 1.53(9 \mathrm{H}, \mathrm{s}) ;{ }^{13} \mathrm{C} \mathrm{NMR}\left(100 \mathrm{MHz}, \mathrm{CDCl}_{3}\right) \delta 167.0(\mathrm{C}), 161.4(\mathrm{C})$, $159.6(\mathrm{C}), 139.5(\mathrm{CH}), 125.2(\mathrm{C}), 121.2(\mathrm{CH}), 82.4(\mathrm{C}), 29.0(\mathrm{Me}), 25.8(\mathrm{Me}), 25.5(\mathrm{Me})$; MS (APcI) $\mathrm{m} / \mathrm{z}$ (relative intensity) $208\left(\mathrm{MH}^{+}, 5 \%\right), 182(5), 153(9), 152(100), 84(5)$.

\footnotetext{
${ }^{3}$ Kochetkov, N. K.; Khomutova, E. D.; Likhosherstov, L. M. Zh. Obshch. Khim. 1959, 29, 1657.

${ }^{4}$ Gräf, E.; Troschütz, R. Synthesis 1999, 1216.
} 
tert-Butyl 2,6-Dimethyl-4-ethylpyridine-3-carboxylate (15cb). The title compound ${ }^{2}$ was obtained as a pale yellow oil (Found: $\mathrm{MH}^{+}, 236.1650 . \mathrm{C}_{14} \mathrm{H}_{21} \mathrm{NO}_{2}$ requires $M H, 236.1651$ ); IR (film) $v_{\max } 2974$, 2932, 1721, 1549, 1562, 1162, 1092; ${ }^{1} \mathrm{H}$ NMR (400 MHz, $\left.\mathrm{CDCl}_{3}\right) \delta 6.83(1 \mathrm{H}, \mathrm{s}), 2.54(2 \mathrm{H}, \mathrm{q}, J$ 7.6), $2.45(3 \mathrm{H}, \mathrm{s}), 2.44(3 \mathrm{H}, \mathrm{s}), 1.53(9 \mathrm{H}, \mathrm{s}), 1.14\left(3 \mathrm{H}, \mathrm{t}, J\right.$ 7.6); ${ }^{13} \mathrm{C} \mathrm{NMR}\left(100 \mathrm{MHz}, \mathrm{CDCl}_{3}\right) \delta 171.2(\mathrm{C})$, $160.7(\mathrm{C}), 156.5(\mathrm{C}), 152.6(\mathrm{C}), 130.4(\mathrm{C}), 123.1(\mathrm{CH}), 85.0(\mathrm{C}), 30.8(\mathrm{Me}), 28.8\left(\mathrm{CH}_{2}\right), 27.1(\mathrm{Me})$, $25.4(\mathrm{Me}), 17.4(\mathrm{Me})$; MS (APcI) $m / z$ (relative intensity) $236\left(\mathrm{MH}^{+}, 100 \%\right), 181$ (5), $180(60)$.

tert-Butyl 2,6-dimethyl-4-phenylpyridine-3-carboxylate (15cc). The title compound ${ }^{2}$ was obtained as a pale yellow oil (Found: $\mathrm{MH}^{+}, 284.1650 . \mathrm{C}_{18} \mathrm{H}_{21} \mathrm{NO}_{3}$ requires $M H$, 284.1647); IR (film) $v_{\max } 2976$, 2928, 1722, 1588, 1551, 1138, 1089, 1031; ${ }^{1} \mathrm{H}$ NMR (400 MHz, $\left.\mathrm{CDCl}_{3}\right) \delta 7.32$ (5H), $6.91(1 \mathrm{H}, \mathrm{s}), 2.53$ $(3 \mathrm{H}, \mathrm{s}), 2.50(3 \mathrm{H}, \mathrm{s}), 1.22(9 \mathrm{H}, \mathrm{s}) ;{ }^{13} \mathrm{C} \mathrm{NMR}\left(100 \mathrm{MHz}, \mathrm{CDCl}_{3}\right) \delta 168.4(\mathrm{C}), 158.4(\mathrm{C}), 155.0(\mathrm{C})$, $148.4(\mathrm{C}), 139.2(\mathrm{C}), 128.7(\mathrm{CH}), 128.7(\mathrm{CH}), 128.6(\mathrm{CH}), 127.5(\mathrm{C}), 121.5(\mathrm{CH}), 82.5(\mathrm{C}), 28.1(\mathrm{Me})$, $24.8(\mathrm{Me}), 23.2(\mathrm{Me})$; MS (APcI) $m / z$ (relative intensity) $284\left(\mathrm{MH}^{+}, 100 \%\right), 250$ (1).

tert-Butyl 2-methyl-6-phenylpyridine-3-carboxylate (15cd). The title compound was obtained as a colorless oil (Found: $\mathrm{MH}^{+}, 270.1487 . \mathrm{C}_{17} \mathrm{H}_{19} \mathrm{NO}_{2}$ requires $M H, 270.1489$ ); IR (film) v $v_{\max } 3006,2980$, 2933, 1699, 1580, 1560, 1446, 1384, 1371, 1305, 1259, 1172, 1146, 1102, 840, 760, 688; ${ }^{1} \mathrm{H}$ NMR $\left(400 \mathrm{MHz}, \mathrm{CDCl}_{3}\right) \delta 8.04(1 \mathrm{H}, \mathrm{d}, J$ 8.2), $7.91(2 \mathrm{H}, \mathrm{m}), 7.44(1 \mathrm{H}, \mathrm{d}, J$ 8.2), $7.29(3 \mathrm{H}), 2.77(3 \mathrm{H}, \mathrm{s}), 1.49$ $(9 \mathrm{H}, \mathrm{s}) ;{ }^{13} \mathrm{C} \mathrm{NMR}\left(100 \mathrm{MHz}, \mathrm{CDCl}_{3}\right) \delta 166.1(\mathrm{C}), 159.4(\mathrm{C}), 158.6(\mathrm{C}), 139.2(\mathrm{CH}), 138.6(\mathrm{C}), 129.5$ $(\mathrm{CH}), 128.8(\mathrm{CH}), 127.3(\mathrm{CH}), 125.3(\mathrm{C}), 117.4(\mathrm{CH}), 81.7(\mathrm{C}), 28.3(\mathrm{Me}), 25.4(\mathrm{Me})$; MS (APcI) m/z (relative intensity) $270\left(\mathrm{MH}^{+}, 90 \%\right), 214$ (100).

2-Methyl-6-phenylpyridine-3-carboxamide (15dd). The title compound was obtained as a pale yellow solid, mp 179-180 ${ }^{\circ} \mathrm{C}$ (Found: $\mathrm{MH}^{+}, 213.1024 . \mathrm{C}_{13} \mathrm{H}_{12} \mathrm{~N}_{2} \mathrm{O}$ requires $M H^{+}, 213.1022$ ); IR (nujol) $\nu_{\max } 1727,1584,1559,1263,1182,1147,1092,1072,1027,841,750 ;{ }^{1} \mathrm{H}$ NMR $\left(400 \mathrm{MHz}, \mathrm{CDCl}_{3}\right) \delta$ $7.95(2 \mathrm{H}, \mathrm{m}), 7.78(1 \mathrm{H}, \mathrm{d}, J 8.1), 7.54(1 \mathrm{H}, \mathrm{d}, J 8.1), 7.41(3 \mathrm{H}), 5.74(2 \mathrm{H}, \mathrm{bs}), 2.75(3 \mathrm{H}, \mathrm{s}) ;{ }^{13} \mathrm{C} \mathrm{NMR}$ $\left(100 \mathrm{MHz}, \mathrm{CDCl}_{3}\right) \delta 170.9(\mathrm{C}), 158.2(\mathrm{C}), 156.5(\mathrm{C}), 138.6(\mathrm{C}), 136.0(\mathrm{CH}), 129.5(\mathrm{CH}), 128.8(\mathrm{CH})$, $128.5(\mathrm{C}), 127.1(\mathrm{CH}), 117.4(\mathrm{CH}), 23.7(\mathrm{Me})$; MS (APcI) $m / z$ (relative intensity) $213\left(\mathrm{MH}^{+}, 100 \%\right)$. 
${ }^{1}$ H NMR Spectra for the Synthesis of Dimethyl Sulfomycinamate (5)

Ethyl 2-(2-Propenyl)oxazole-4-carboxylate (25)

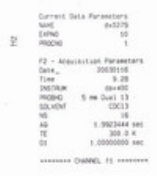

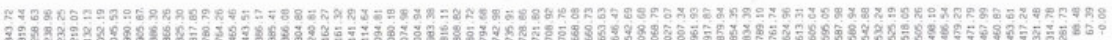
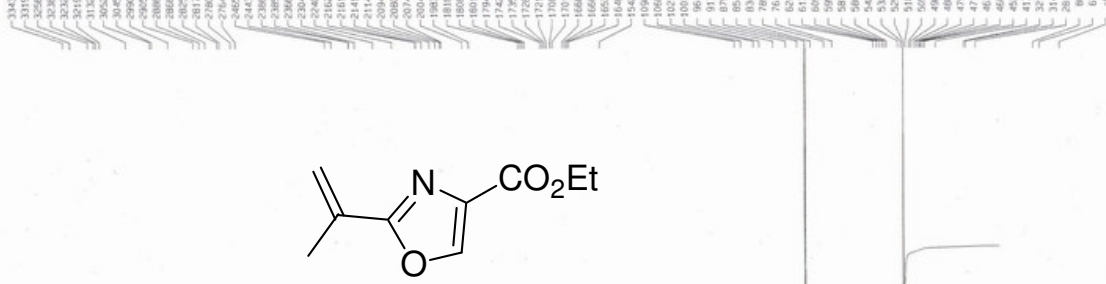

25

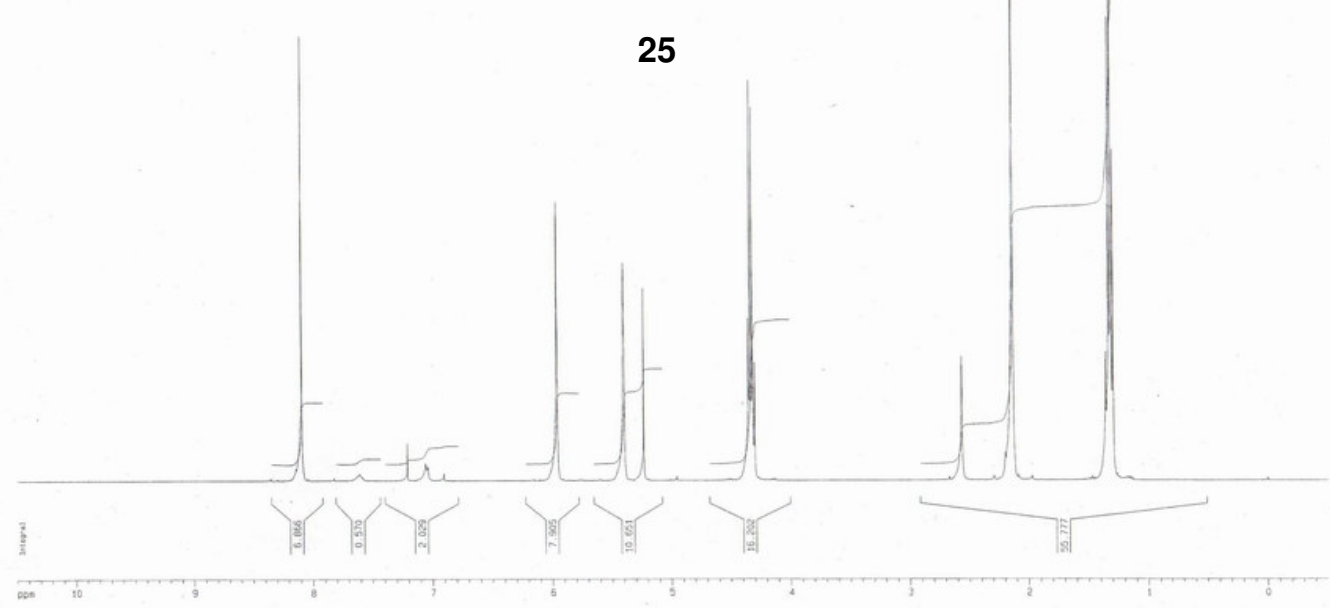


2-(2-Propenyl)oxazole-4-carboxylic acid (26)
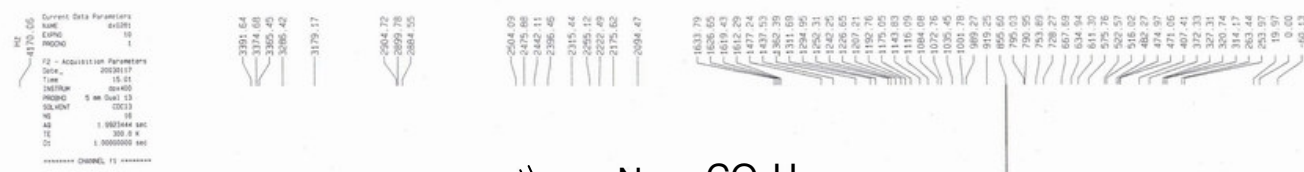<smiles>C=C(C)c1nc(C(=O)O)co1</smiles>

26

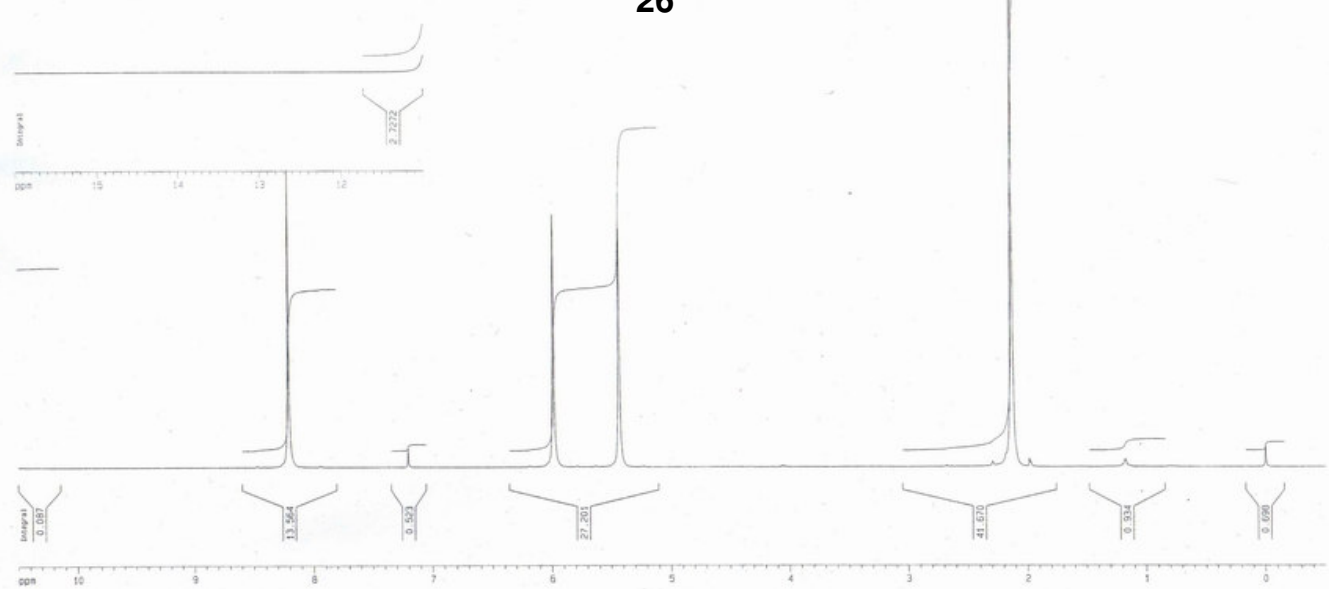

S-Ethyl 3-Hydroxy-3-[2-(2-propenyl)oxazol-4-yl]thiopropenoate (27) (+ tautomer)

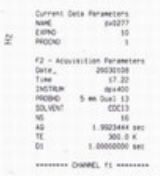

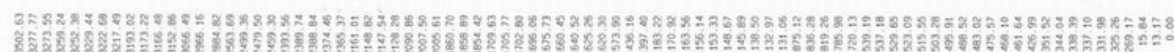

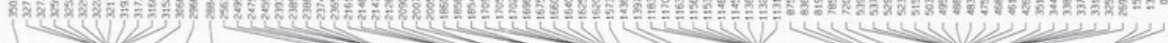<smiles>C=C(C)c1nc(/C(O)=C/C(=O)SCC)co1</smiles>
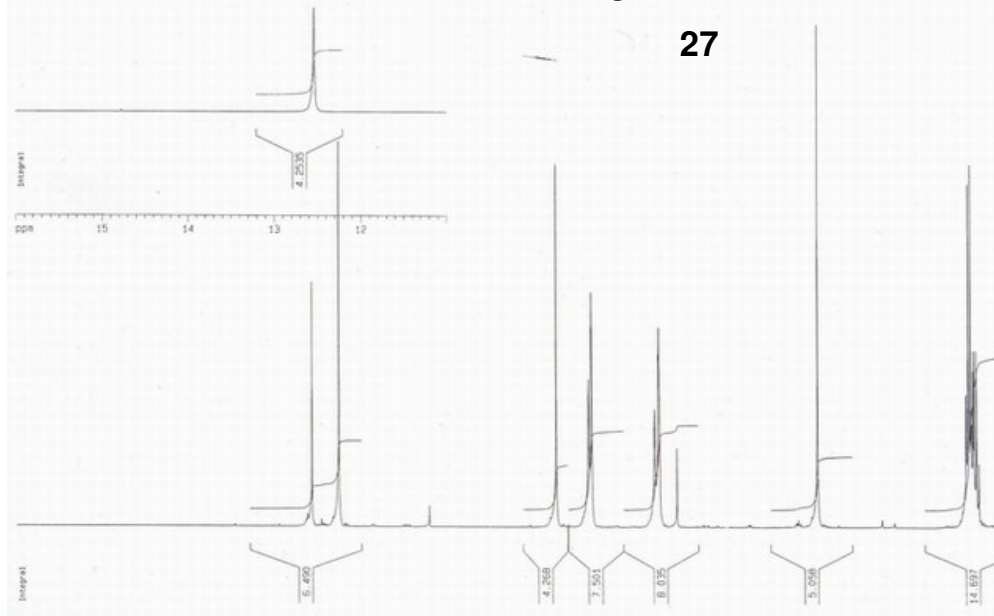
(S)- $N$-\{3-Oxo-3-[2-(2-propenyl)oxazol-4-yl]propanoyl\}-O-tert-butylserine Methyl Ester $(+$ tautomer)
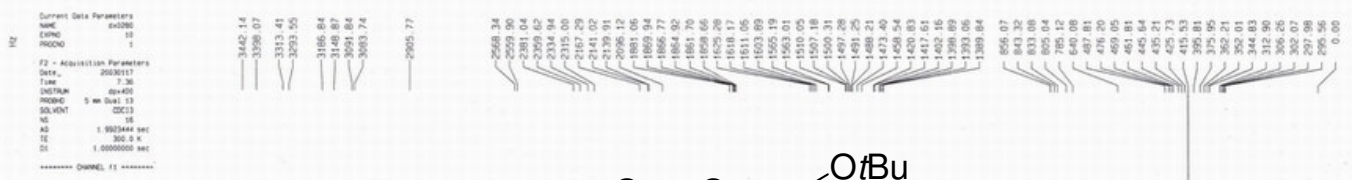<smiles>C=C(C)c1nc(C(=O)CC(=O)NC(COCC)C(C)=O)co1</smiles>

$7 a$
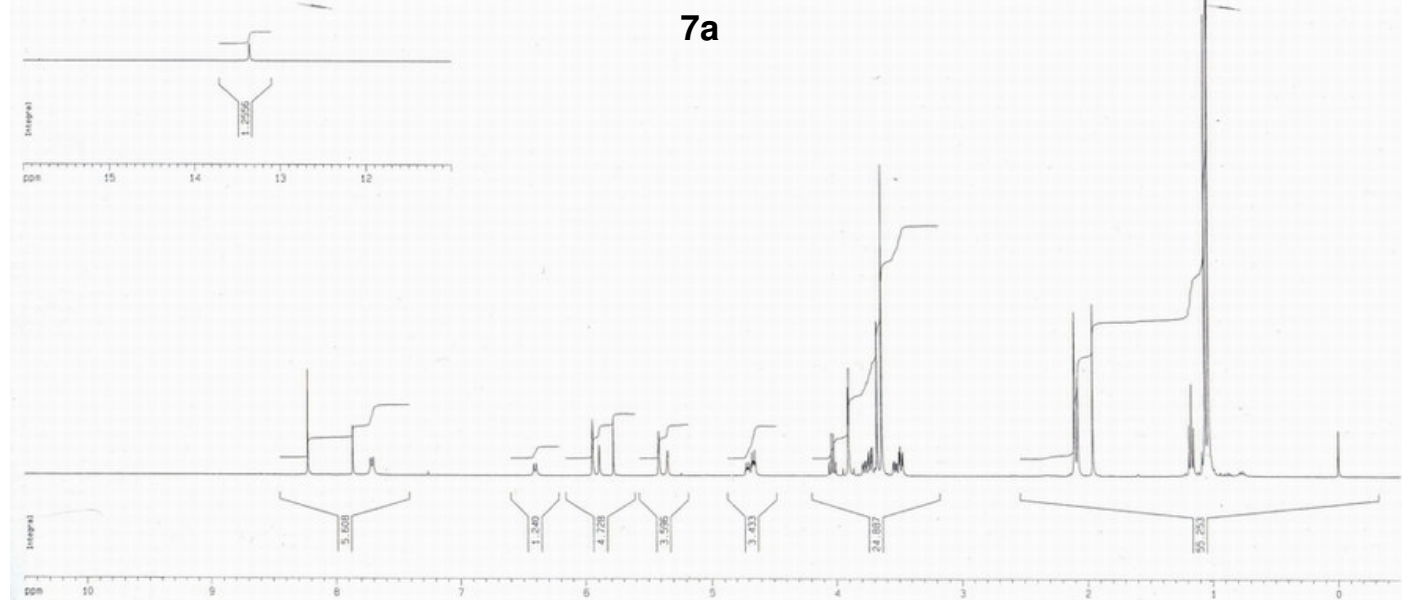

(S)- $N$-\{3-Amino-3-[2-(2-propenyl)oxazol-4-yl]propenoyl\}-O-tert-butylserine Methyl Ester (8a)
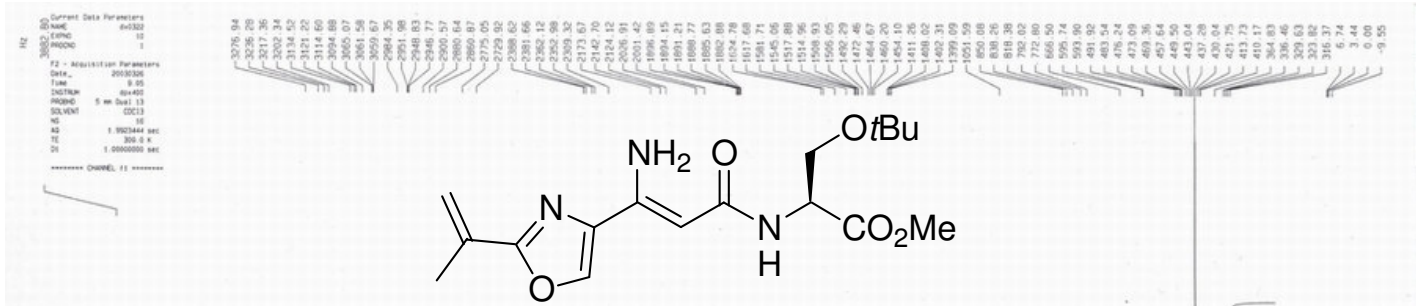

$8 a$

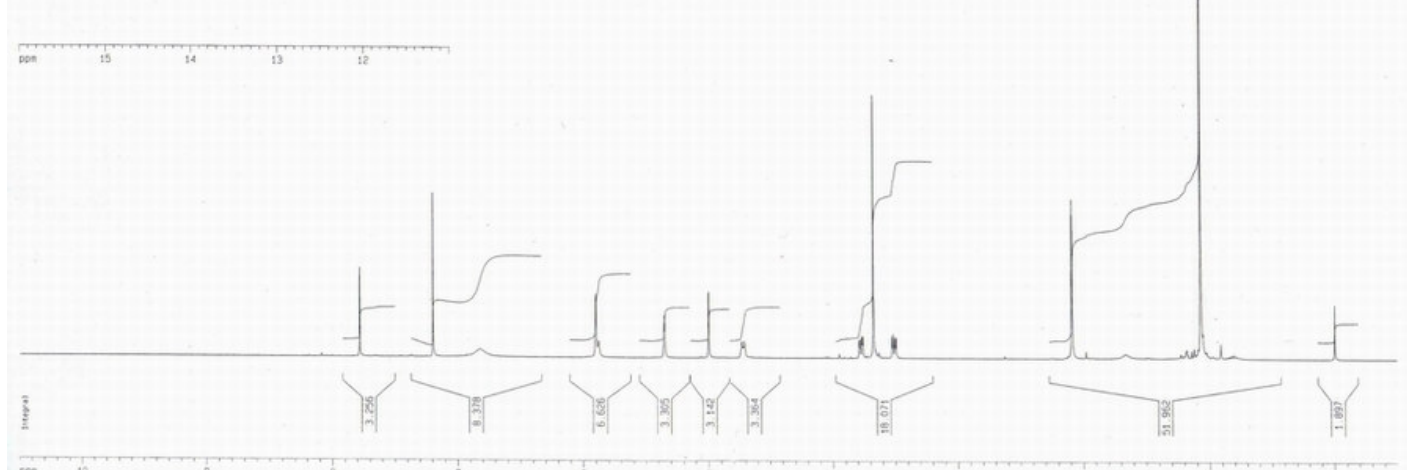


(S)-O-tert-Butyl- $N$-(\{2-[2-(2-propenyl)oxazol-4-yl]-6-methoxycarbonylpyridin-3yl\}carbonyl)serine Methyl Ester (10a)

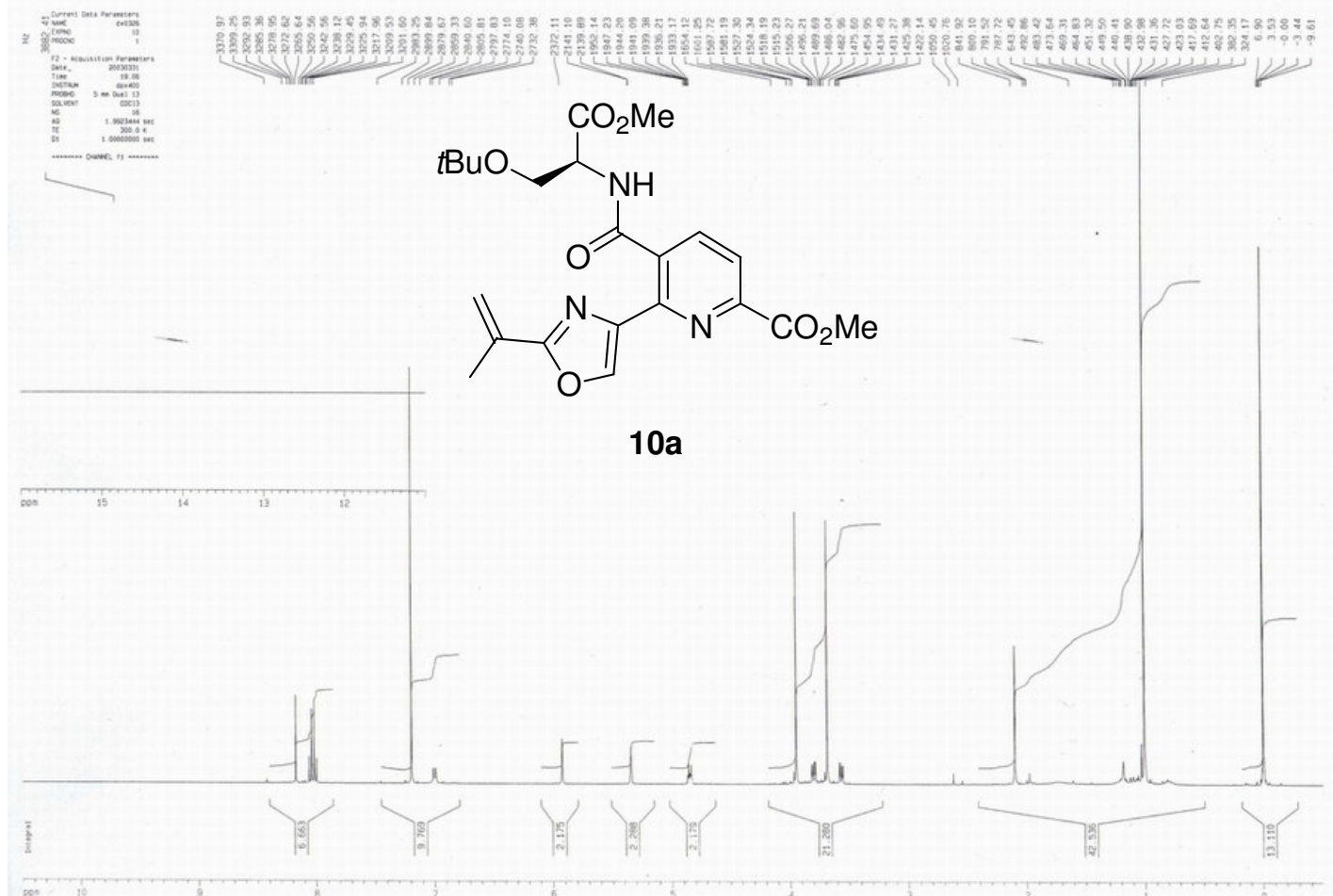

(S)-N-(\{2-[2-(2-Propenyl)oxazol-4-yl]-6-methoxycarbonylpyridin-3-yl\}carbonyl)serine Methyl Ester (28)
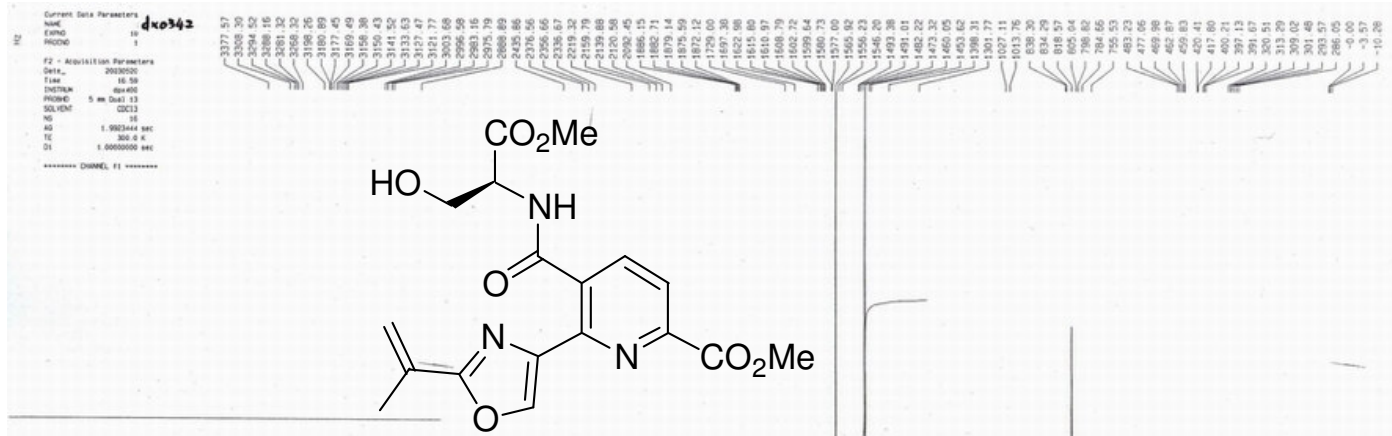

28

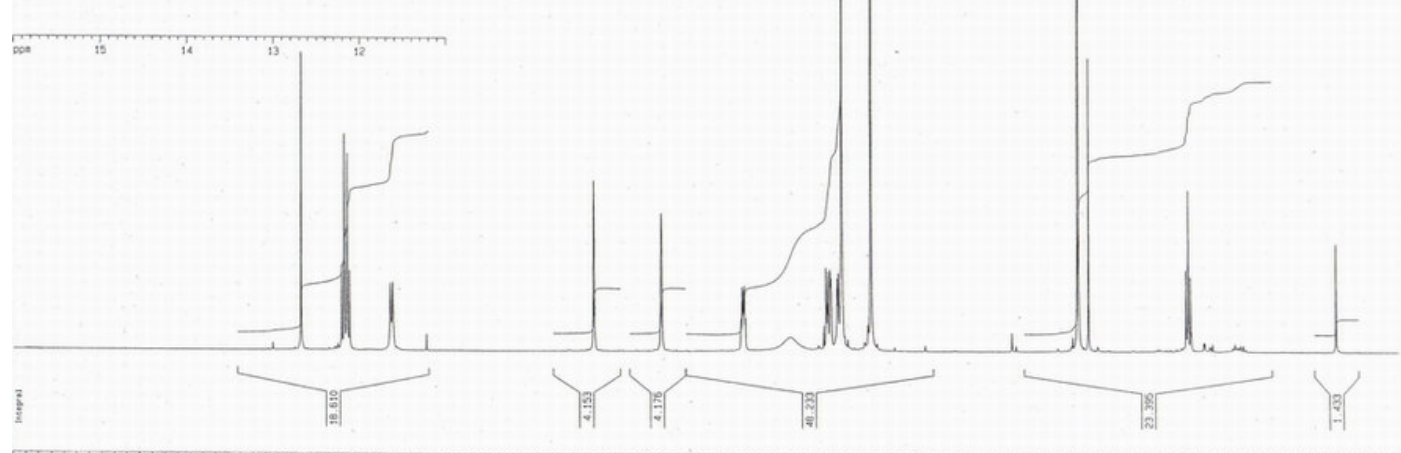


(S)-Methyl 2-[2-(2-Propenyl)oxazol-4-yl]-3-(4-methoxycarbonyl-2-oxazolin-2-yl)pyridine-6carboxylate (29)

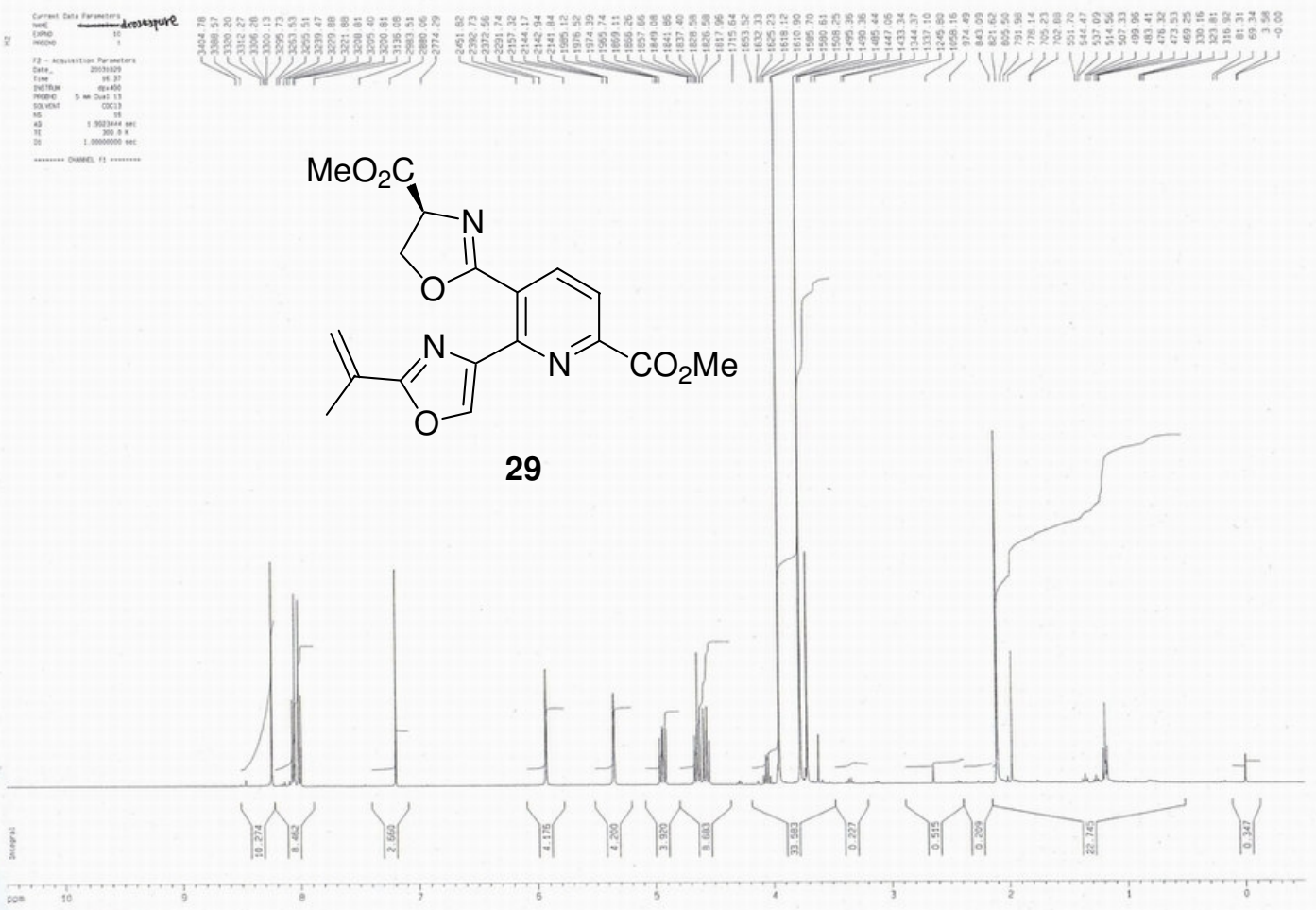

(S)-N-(\{2-[2-(2-Propenyl)oxazol-4-yl]-6-methoxycarbonylpyridin-3-yl $\}$ thiocarbonyl)serine Methyl Ester (30)

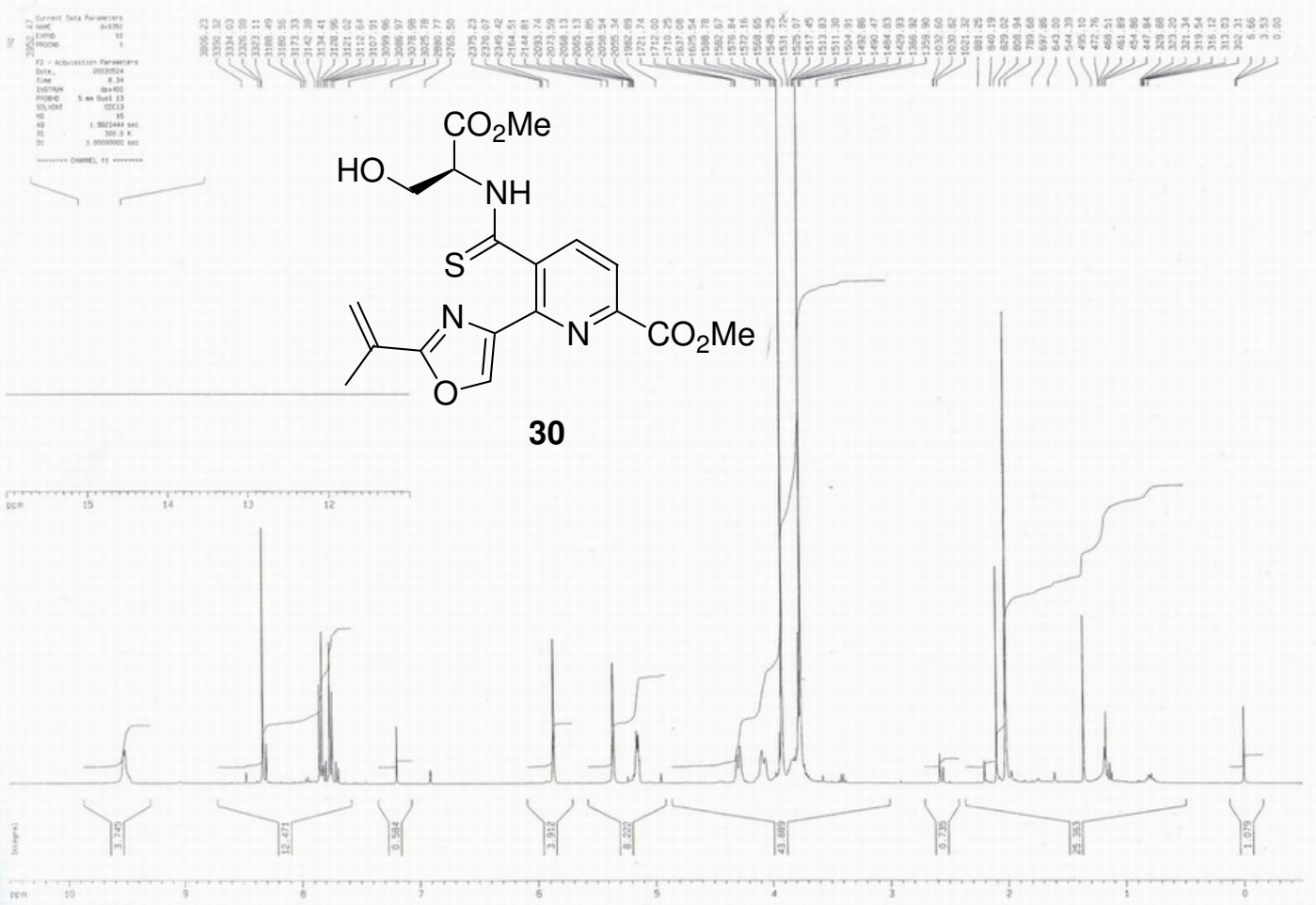


(R)-Methyl 2-[2-(2-Propenyl)oxazol-4-yl]-3-(4-methoxycarbonyl-2-thiazolin-2-yl)pyridine-6carboxylate (31)

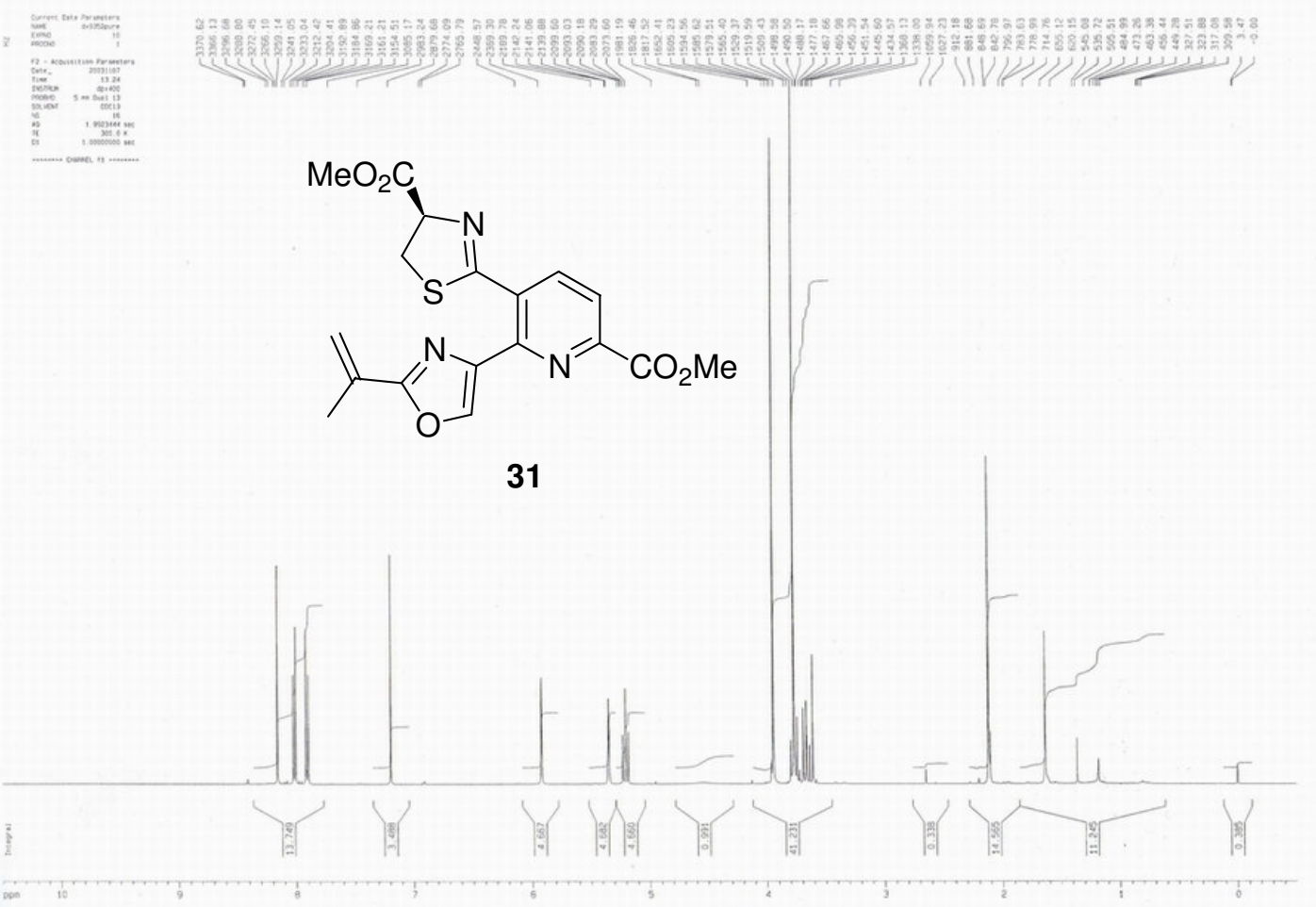

Methyl carboxylate (32)

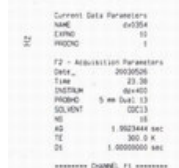

2-[2-(2-Propenyl)oxazol-4-yl]-3-[4-(methoxycarbonyl)thiazol-2-yl]pyridine-6-

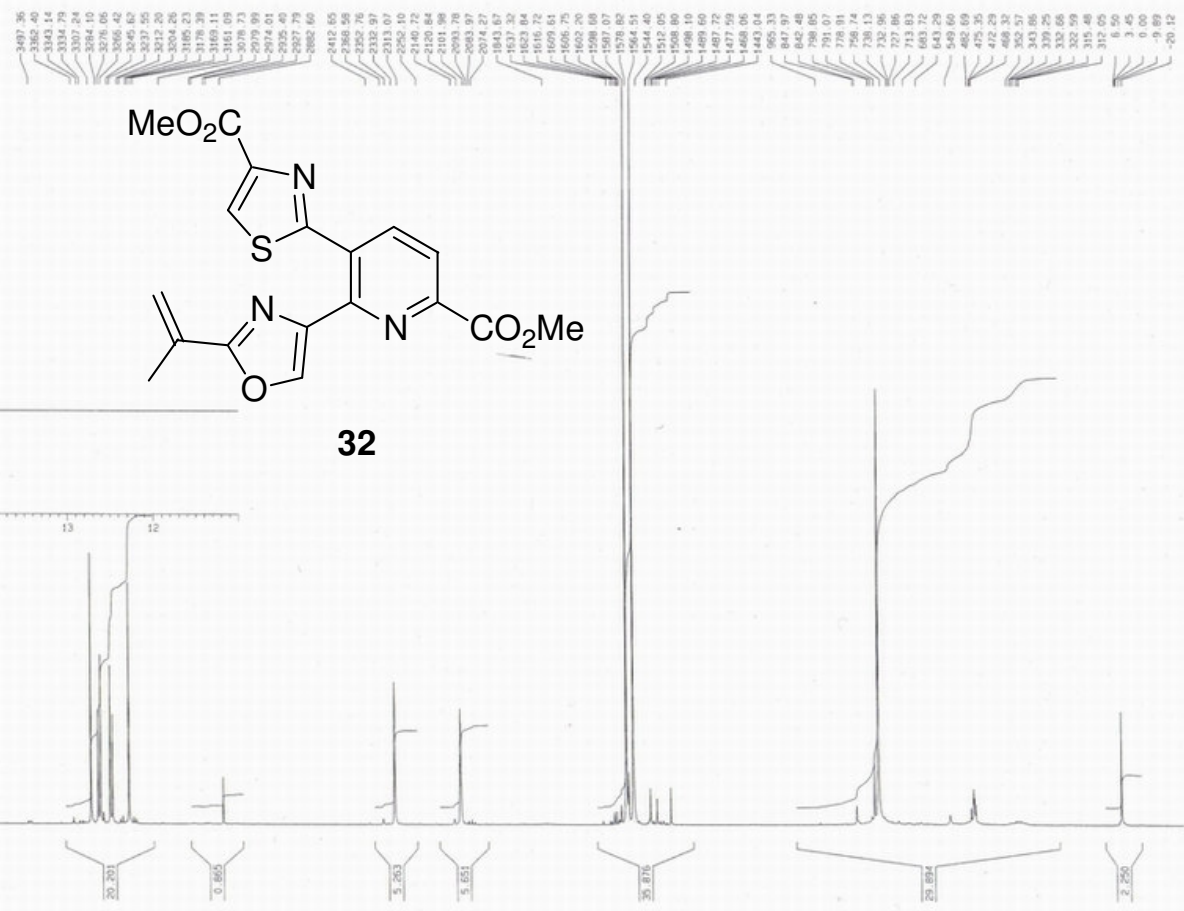




\section{$\underline{{ }^{1} \mathrm{H} \text { NMR spectrum }\left(400 \mathrm{MHz}, \mathrm{CDCl}_{3}\right) \text { of dimethyl sulfomycinamate (5) }}$}
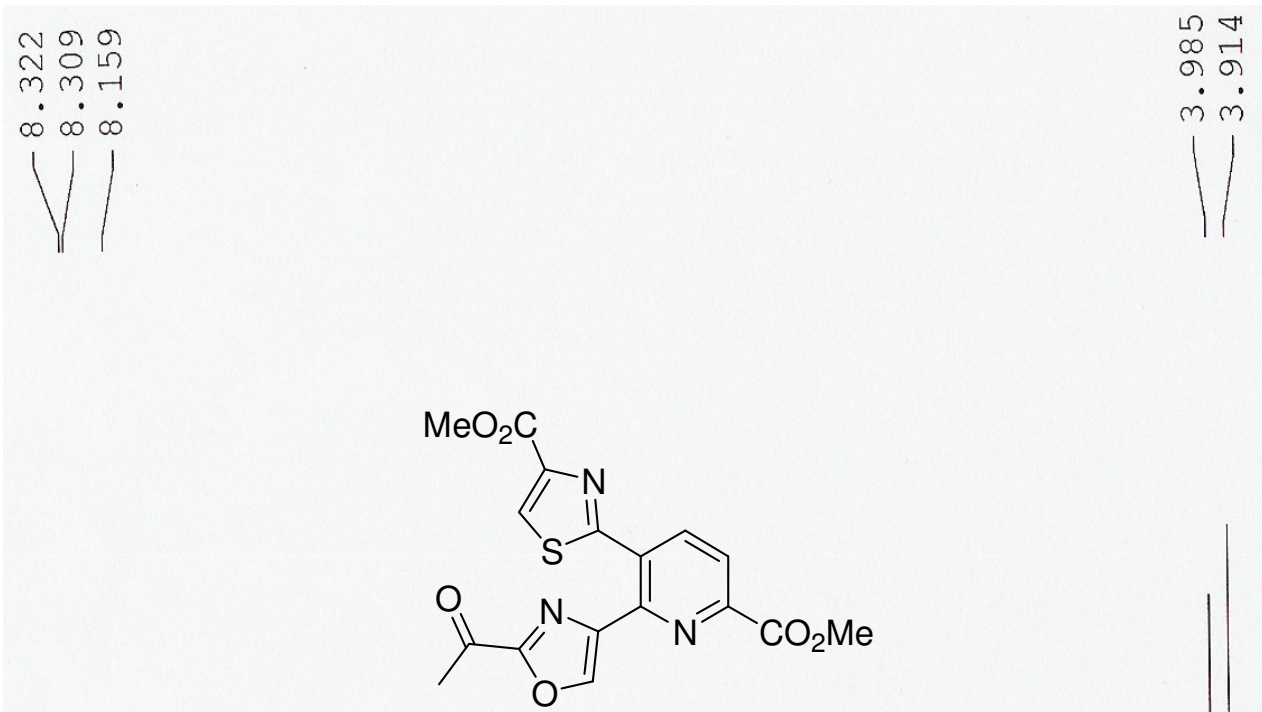

dimethyl sulfomycinamate (5)

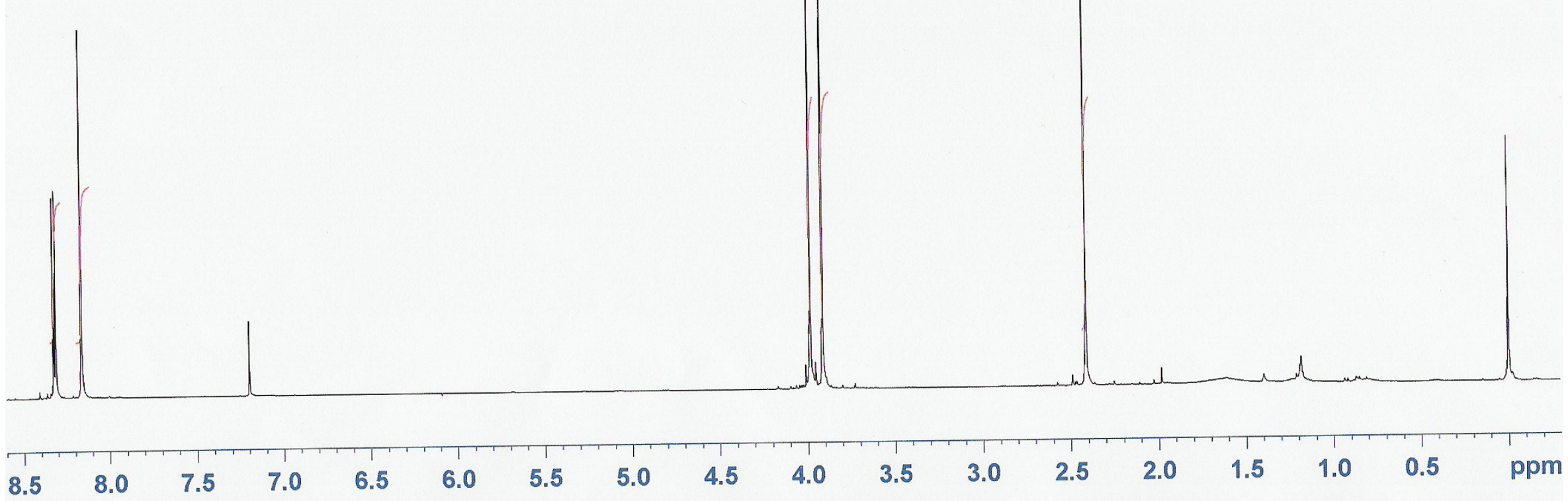


${ }^{13} \mathrm{C}$ NMR spectrum $\left(100 \mathrm{MHz}, \mathrm{CDCl}_{3}\right)$ of dimethyl sulfomycinamate (5)
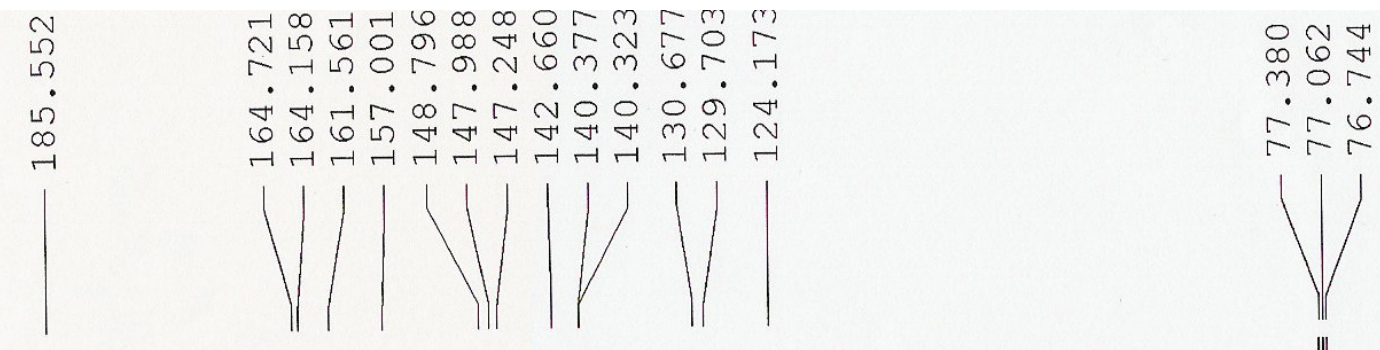

ลู่

$\dot{m} \dot{ }$

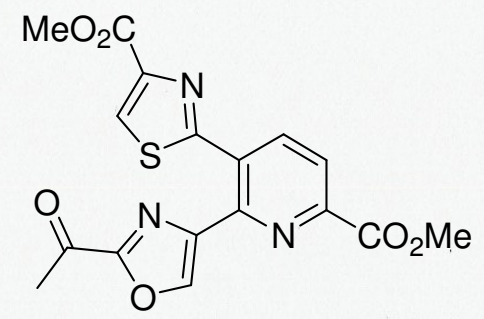

dimethyl sulfomycinamate (5)

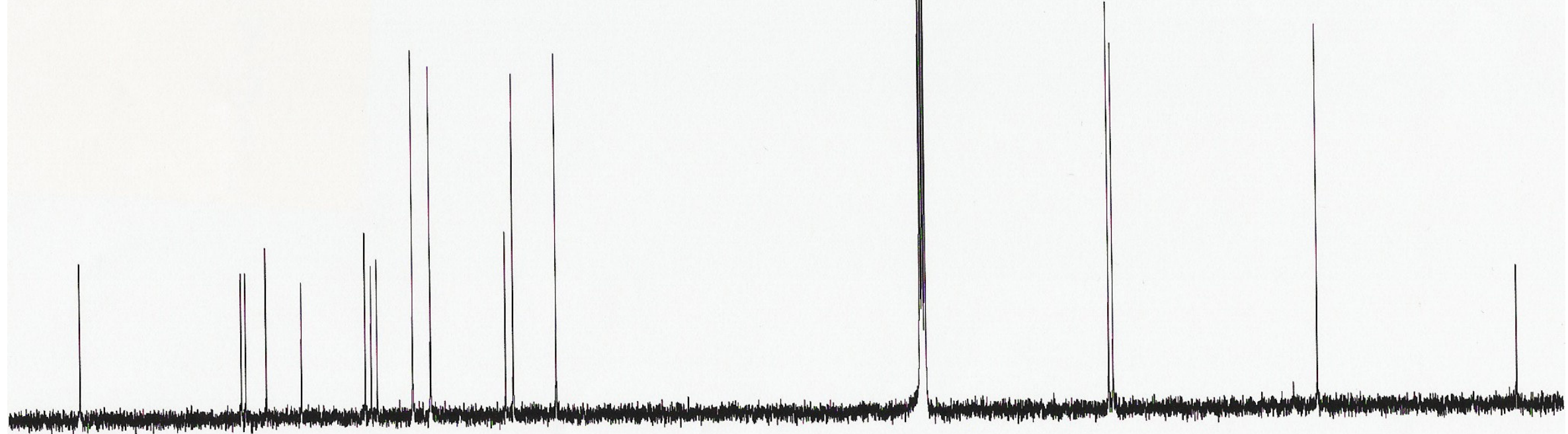

